\title{
Evaluation of a multi-component community tobacco intervention in three remote Australian Aboriginal communities
}

\begin{abstract}
Objectives: To assess the effect of community tobacco interventions in Aboriginal communities.

Methods: The study consisted of a preand post-study of the effect of a multicomponent tobacco intervention conducted in six Aboriginal communities in the Northern Territory (NT). The intervention included sports sponsorship, health promotion campaigns, training health professionals in the delivery of smoking cessation advice, school education about tobacco, and policy on smoke-free public places. The study was conducted in three intervention communities and three matched control communities. Surveys were used to measure changes in prevalence of tobacco use, changes in knowledge, and attitudes to cessation in intervention communities.
\end{abstract}

Results: Tobacco consumption decreased in one intervention community compared with the matched control community; the trends of consumption (as measured by tobacco ordered through points of sale) in these communities were significantly different $(t=-4.5,95 \% \mathrm{Cl}-33.6$ $--12.5, p \leq 0.01)$. Community samples in intervention communities included 920 participants. There was no significant change in the prevalence of tobacco use, although knowledge of the health effects of tobacco and readiness to quit increased.

Conclusions: Although it is difficult to demonstrate a reduction in tobacco consumption or in the prevalence of tobacco use as a result of multi-component community tobacco interventions delivered in Aboriginal communities, such interventions can increase awareness of the health effects of tobacco and increase reported readiness to cease tobacco use.

(Aust N Z J Public Health 2006; 30: 132-6)

\author{
Rowena G. Ivers \\ Menzies School of Health Research, Northern Territory \\ Anthony Castro, David Parfitt \\ Tobacco Action Project, Department of Health and Community Services \\ (previously Territory Health Services), Northern Territory
}

\author{
Ross S. Bailie \\ Menzies School of Health Research, Northern Territory
}

Peter H. D'Abbs

School of Public Health and Tropical Medicine, James Cook University, Queensland

\author{
Robyn L. Richmond \\ School of Public Health and Community Medicine, University of New South Wales
}

$\mathbf{T}$ here has been little evaluation of interventions designed to reduce the harm resulting from tobacco use for Aboriginal Australians. ${ }^{1}$ There is evidence that community tobacco interventions are effective in reducing uptake of tobacco use in young people. ${ }^{2}$ The effectiveness is less clear for interventions for adults. In one large trial conducted in the United States, the Community Intervention Trial for Smoking Cessation (COMMIT), a large-scale multicomponent community intervention was assessed to ascertain the effect on the prevalence of smoking. Eleven matched pairs of communities were randomly assigned to either a control or intervention group, with the intervention group exposed to a multicomponent intervention delivered through the media, by health care providers, at worksites and through cessation programs. A cohort of smokers followed over the trial showed a significant increase in the cessation rate among light-moderate smokers in intervention communities compared with that in control communities ( $31 \%$ vs. $28 \%$, $p \leq 0.01)$ and a non-significant decrease in the cessation rate in heavy smokers in the intervention communities compared with that in the control communities (18\% vs. 19\%). ${ }^{3}$ COMMIT demonstrated an overall drop in the prevalence of smoking of $3.5 \%$ in intervention communities and a 3.2\% drop in control communities, a non-significant difference. $^{4}$

In a randomised controlled trial of a community action intervention to address smoking behaviour in rural towns in New South Wales, adults in towns participating in the intervention showed mainly nonsignificant increases in quit rate and nonsignificant decreases in uptake rate compared with control towns. ${ }^{5}$ Significantly more male smokers (7\%) quit in towns participating in the intervention than in the control towns.
Submitted: May 2005

Revision requested: September 2005

Accepted: January 2006

\section{Correspondence to:}

Ms Rowena Ivers, Illawarra Aboriginal Medical Service, PO Box 1161, South Coast Mail Centre, New South Wales 2521. Fax (02) 4228 6153; e-mail: Rowenalvers@ bigpond.com.au 
Media campaigns have an effect in reducing smoking prevalence. The National Tobacco Campaign, launched in 1997, involved a media campaign (including television and radio advertising) and co-ordination with Quitline services, and resulted in a statistically significant reduction of $1.5 \%$ in the estimated adult prevalence of smoking. ${ }^{6}$ The campaign resulted in an increase in the population cessation rate from $8 \%$ to $11 \%{ }^{6}$ There were no differences in awareness or message recall for Aboriginal people and the general population. ${ }^{6}$ The effect of community tobacco programs has otherwise not been assessed for Aboriginal or Torres Strait Islander people.

\section{Objectives}

To assess the impact of multi-component, community-based tobacco interventions in remote Aboriginal communities.

\section{Methods}

\section{Setting}

The study was conducted in six remote Aboriginal communities in the Northern Territory. The Northern Territory Department of Health and Community Services, then known as Territory Health Services (THS), sponsored the Tobacco Action Project, which offered small grants in 1999/2000 to reduce the damaging effects of tobacco for Aboriginal people. The study involved comparison of tobacco consumption in three communities that were successful in their application for a grant for a tobacco project to that in three control communities. The three control communities were matched so as to be of similar geographical location, culture and socio-economic status $^{7}$ to the intervention communities; matched communities also had the same level of health service (for example, all communities only had visiting doctors). Control communities were also selected to ensure that mode of tobacco use was similar to that of intervention communities; tobacco was mainly consumed as tailor-made cigarettes or 'rollies' of loose tobacco in all communities. It was occasionally smoked in a pipe (commercial, crab-claw or Macassan (Indonesian) pipe) or chewed with eucalyptus ash ('mubbudge'). Retail sources of tobacco in the control and intervention communities were similar (that is, tobacco was almost exclusively bought from a single community store). Community A had a population of 477 people, and Control Community A had a population of 282 people. ${ }^{7}$ Community B had a population of 266 people and Control Community B had a population of 384 people. ${ }^{7}$ Community C had a population of 939 people and Control Community $\mathrm{C}$ had a population of 478 people. ${ }^{7}$

\section{The intervention}

The multi-component intervention included interventions developed by the community and delivered in conjunction with a range of evidence-based tobacco interventions, which were delivered by Aboriginal project officers. Community interventions included the introduction of smoke-free enclosed public places in Community A, a Women's Centre tobacco education program in Community B and sports carnival sponsorship in Community $\mathrm{C}$ (each lasting three to five days). Evidence-based interventions included: training all health professionals in delivering a brief intervention on tobacco, involving brief advice on cessation with culturally appropriate health promotion materials (pamphlets, posters and flip charts) previously developed in the region with Aboriginal communities; use of nicotine patches; a point-of-sale intervention; and school education about tobacco, delivered over a period of months. Evidence-based interventions were delivered by the Aboriginal project officers.

No tobacco interventions were carried out in control communities during the intervention year.

\section{Measurement of tobacco consumption}

The measurement of tobacco turnover has successfully been used in previous studies in other populations ${ }^{8}$ and in Indigenous communities. ${ }^{9,10}$ Tobacco consumption was measured over a 14month period in the six participating communities by accessing data from tobacco vendors. Information on monthly orders for each type of cigarette and tobacco was collected from either the store manager or from the store's wholesaler for each of the communities in the study. The data were entered into a database and analysed using the statistical computer program Stata ${ }^{11}$ to compare changes in consumption in intervention and control communities. To calculate the amount of tobacco in each 'rollie', a small sample of people who regularly smoked loose tobacco were each asked to roll a 'rollie'. Each 'rollie' was weighed and the weights were averaged so as to compare with the amount of tobacco in a standard cigarette (henceforth called a 'cigarette equivalent'). Consumption was then calculated using the number of cigarettes or 'cigarette-equivalents' of loose tobacco ordered through stores for each month; this was then divided by the number of community members aged 12 years or older, as estimated from the 1996 Australian Bureau of Statistics Census.

\section{Analysis of tobacco consumption}

Consumption was graphed to show rolling averages of tobacco orders for each three-month period. The researcher modelled the difference in trend in tobacco consumption between intervention and control communities with linear regression, using an interaction term for trend in tobacco consumption over time. Data on price were sought from the vendor with the highest volume of sales in each community.

\section{Community surveys}

The evaluation of the project also involved pre and post evaluation in the intervention communities, at baseline - in the month prior to the planned intervention - and at a follow-up visit, a year later. The evaluation included community-wide surveys to identify any changes in smoking behaviour, attitudes (readiness to quit), and knowledge following the community tobacco interventions. Indigenous research assistants assisted the researcher (RI) with recruiting participants with the aim of attaining a comprehensive sample, explained the nature of the 
research project (in local language or in English), and obtained informed consent from participants. Participants were followed up using a similar questionnaire, one year later. At the second visit, they were also offered information about tobacco by the researcher or local research assistant, including advice about cessation.

\section{Analysis of community surveys}

The data from community surveys were analysed using Stata ${ }^{11}$ to assess changes in smoking behaviour and attitudes to cessation (readiness to quit) in a cohort of those who participated in both the baseline and follow-up surveys, and a cross-sectional sample, including all participants who participated in one or both surveys.

\section{Results}

\section{Tobacco consumption}

Orders of cigarette equivalents per person over the age of 12 years in Community A fell over the intervention year but rose at Control Community A. Tobacco orders for these communities were compared by assessing differences in the trend of the line of best fit for tobacco orders for each community over the 14-month period. The trend of tobacco orders in Community A declined significantly more than that of tobacco orders in Control Community A ( $t=-4.5$, $95 \%$ CI $-33.6--12.5, p \leq 0.01)$.

At baseline, orders of cigarette equivalents per head of population were higher at Community B than at Control Community B. Orders of cigarette equivalents per person over the age of 12 years fell in both communities. Data on tobacco orders were not available for some months of the year because of major flooding in Community B. The trends of the decrease in tobacco orders did not differ significantly $(t=-1.7,95 \%$ CI $-28.8-4.9, p=0.13)$.

Orders per person aged 12 or over were substantially higher at Control Community $\mathrm{C}$ than at Community $\mathrm{C}$ at baseline and throughout the intervention year. Orders fell at Community $\mathrm{C}$ over the intervention year and rose at Control Community $\mathrm{C}$, however the trend of tobacco orders in these communities did not differ significantly $(t=-1.2,95 \%$ CI $-13.9-3.8, p=0.25)$.

\section{Community surveys}

Community workers classified 1,228 people as residents of their communities at the baseline visit, of whom 643 participated in the community survey. Of 1,201 residents at the follow-up visit, 628 participated in the community survey. Overall, 920 community members from intervention communities participated in one or both of the surveys; $20 \%$ of residents participated in the baseline survey only, $19 \%$ in the follow-up survey only and $24 \%$ participated in both surveys; $37 \%$ did not participate in the study (see Table 1).

Among participants, 91\% were Aboriginal and 9\% were nonAboriginal, $50 \%$ were male and $15 \%$ were aged $<18$ years, $46 \%$ aged 18-34 years and 39\% aged $\geq 35$ years. Five of those included in the baseline survey passed away during the intervention year; four were smokers. Those lost to follow-up were more likely to be non-Aboriginal $(p<0.01)$ and less likely to be ready to quit $(p=0.03)$; however, they did not differ on other indices.

Of those interviewed at both baseline and follow-up surveys, $13(29 \%)$ of those who claimed to be ex-smokers had given up in the year prior to the baseline survey, and $32(71 \%)$ had given up prior to that. Overall, $10 \%$ of people who claimed to be smokers at the baseline visit had quit at the follow-up survey. However, some ex-smokers (11 participants, 24\% of ex-smokers) and some who reported that they had never smoked (11 participants, $16 \%$ of never-smokers) took up smoking during the intervention year. Of those who participated in both surveys, there was a net gain of 11 smokers in intervention communities at the end of the intervention year. The prevalence of smoking in those who participated in both surveys in intervention communities rose from $68 \%$ to $70 \%$, however this rise was not significant (McNemar's test statistic $=0.64, p=0.52$ ) (see Table 2).

When data were assessed for all participants (that is, who participated in one or both surveys - a cross-sectional sample), the prevalence of tobacco use dropped from $68 \%$ at the baseline to $67 \%$ at the follow-up survey.

Of participants interviewed in both surveys, significantly more smokers reported that they were light smokers $(<70$ cigarettes per week) at the follow-up survey than at the baseline survey ( $40 \%$ vs. $46 \%$, McNemar's test statistic $=5.2, p=0.03$ ). Those who smoked

Table 1: Participation of residents in surveys, intervention communities.

\begin{tabular}{|c|c|c|}
\hline $\begin{array}{l}\text { Participation } \\
\text { in surveys }\end{array}$ & $\begin{array}{l}\text { Residents who } \\
\text { participated in } \\
\text { surveys }\end{array}$ & $\begin{array}{l}\text { Residents who } \\
\text { participated in } \\
\text { surveys (\%) }\end{array}$ \\
\hline \multicolumn{3}{|l|}{$\overline{\text { Community A }}$} \\
\hline Baseline survey only & 98 & 22 \\
\hline Follow-up survey only & 91 & 21 \\
\hline Both surveys & 140 & 32 \\
\hline Neither survey & 115 & 26 \\
\hline Total & 444 & $100^{\mathrm{a}}$ \\
\hline \multicolumn{3}{|l|}{ Community B } \\
\hline Baseline survey only & 105 & 28 \\
\hline Follow-up survey only & 88 & 23 \\
\hline Both surveys & 132 & 35 \\
\hline Neither survey & 50 & 13 \\
\hline Total & 375 & $100^{\mathrm{a}}$ \\
\hline \multicolumn{3}{|l|}{ Community C } \\
\hline Baseline survey only & 89 & 14 \\
\hline Follow-up survey only & 98 & 15 \\
\hline Both surveys & 79 & 12 \\
\hline Neither survey & 373 & 58 \\
\hline Total & 639 & $100^{a}$ \\
\hline \multicolumn{3}{|l|}{ All communities } \\
\hline Baseline survey only & 292 & 20 \\
\hline Follow-up survey only & 277 & 19 \\
\hline Both surveys & 351 & 24 \\
\hline Neither survey & 538 & 37 \\
\hline Total & 1,458 & 100 \\
\hline
\end{tabular}


$<70$ cigarettes per week (light smokers) were not significantly more likely to quit than those who smoked 70 cigarettes or more per week (moderate and heavy smokers); nine light smokers had quit compared with eight moderate or heavy smokers (10\% vs. $\left.5 \%, \chi^{2}=1.7,1 d f, p=0.19\right)$. Tobacco orders differed from selfreported consumption of tobacco. Store data showed that 15.8 cigarette equivalents were ordered per day per smoker aged 12 or over, compared with self-reported consumption of 16.9 cigarette equivalents per day, at baseline, and 8.7 vs. 14.0 cigarette equivalents per day at follow-up.

Of those who participated in both surveys, significantly more people were taking action to quit or thinking about quitting following the intervention year (61\% vs. $72 \%$, McNemar's test statistic $=6.22, p=0.02$ ). There was also a significant increase in the number of people who believed that lung cancer was linked to tobacco use ( $85 \%$ at baseline and $90 \%$ at follow-up, McNemar's test statistic $=4.74, p=0.04)$ and in the number of people who believed that heart disease was linked to tobacco use $(82 \%$ at baseline and $89 \%$ at follow-up, McNemar's test statistic $=8.32$, $p<0.01$ ).

\section{Discussion}

\section{Changes in tobacco consumption}

Over the intervention year, tobacco consumption appeared to decline in all intervention communities. Tobacco consumption rose in two control communities; however, the trend in intervention communities only differed significantly from that of control communities for Community A and Control Community A, with the trend suggesting a non-linear drop in consumption.

We found no decrease in the prevalence of tobacco use from the community surveys (and indeed, a non-significant rise in those surveyed at both the baseline and follow-up surveys). The decrease in consumption appeared to be caused by a decrease in

Table 2: Changes in prevalence of tobacco use, intervention communities.

\begin{tabular}{|c|c|c|c|}
\hline & Smokers (\%) & Non-smokers (\%) & Total (\%) \\
\hline \multicolumn{4}{|c|}{ Community A } \\
\hline Baseline & $91(65)$ & $49(35)$ & $140(100)$ \\
\hline Follow-up & $92(66)$ & $48(34)$ & $140(100)$ \\
\hline Difference & \multicolumn{3}{|c|}{ McNemar's test statistic $=0.11, p=1.0$} \\
\hline \multicolumn{4}{|l|}{ Community B } \\
\hline Baseline & $105(80)$ & $27(20)$ & $132(100)$ \\
\hline Follow-up & $104(79)$ & $28(21)$ & $132(100)$ \\
\hline Difference & \multicolumn{2}{|c|}{ McNemar's $=0.06, p=1.0$} & \\
\hline \multicolumn{4}{|c|}{ Community C } \\
\hline Baseline & $47(59)$ & $32(41)$ & 79 (100) \\
\hline Follow-up & $48(61)$ & $31(39)$ & $79(100)$ \\
\hline Difference & \multicolumn{2}{|c|}{ McNemar's $=0.08, p=1.0$} & \\
\hline \multicolumn{4}{|c|}{ All communities } \\
\hline Baseline & $239(68)$ & $112(32)$ & $351(100)$ \\
\hline Follow-up & $244(70)$ & $107(30)$ & $351(100)$ \\
\hline Difference & \multicolumn{2}{|c|}{ McNemar's $=0.64, p=0.52$} & \\
\hline
\end{tabular}

tobacco consumed by individual smokers - for example, a drop in the number of cigarettes smoked per week.

Other confounding factors, such as anti-tobacco advertising and price rises (which, although not reported here, were measured, and occurred to a similar extent in both control and intervention communities) did not appear to have had a marked effect on consumption.

\section{Changes in smoking behaviour and attitudes}

Our study reports similar findings to other studies in which there was no significant change in the prevalence of tobacco use $^{4,5}$ following community tobacco interventions. It is of some concern that there was a trend towards an increase in the prevalence of tobacco use. This was attributed to a combination of young people taking up smoking and ex-smokers re-initiating smoking. Behaviour change is not likely to occur immediately as a result of community anti-smoking programs, but smokers may be encouraged to quit over a period of years by repeated cessation messages and changes in tobacco culture (for example, less acceptance of smoking in public places).

Although there was no decrease in the prevalence of tobacco use among those who were interviewed at both baseline and follow-up, many $(20 \%)$ reported that they had consumed less tobacco at the end of the intervention year, which supports findings from our data on tobacco orders. Our finding is supported by the NDS Household Survey, ${ }^{12}$ in which $41 \%$ of smokers and $32 \%$ of Indigenous smokers surveyed reported that they had decreased consumption in the previous year. However, decreasing consumption of tobacco is not necessarily linked to improvement in health outcomes. The relative over-reporting of tobacco consumption may also suggest there were other sources of tobacco in these communities that were not accounted for in records of store orders, for example tobacco purchased from regional towns. This implies that it was unlikely that sales of tobacco to non-residents had falsely elevated store orders of tobacco.

At baseline, $61 \%$ of smokers said that they were taking action to quit or were thinking about quitting; over the course of the intervention year this increased significantly to $72 \%$ - this is encouraging. By comparison, in the evaluation of the National Tobacco Campaign, $52 \%$ of smokers considered that they were preparing to quit or were contemplating cessation at the baseline survey, and this increased to $57 \%$ over the course of the campaign. ${ }^{6}$ The low quit rate (even in people who stated they were taking action to quit or thinking about quitting) may have been because there were too many barriers to cessation, particularly that smoking was seen as normal behaviour, even if a smoker was seriously considering cessation. Alternatively, participants may have been polite or willing to please the researcher by stating that they were more interested in quitting than they actually were.

The level of knowledge about health effects of tobacco (lung cancer and heart disease) was high in intervention communities, compared with previous studies in the general Australian population. ${ }^{13}$ Yet, although knowledge about health issues increased over the intervention year, it was not linked to a greater likelihood 
of cessation, again, probably because of the large numbers of barriers to cessation. Barriers to the success of the community tobacco interventions included competing health priorities such as alcohol abuse, insufficient resources to run preventive programs, lack of health promotion staff, and the occurrence of natural phenomena such as cyclones and floods (with one intervention community being evacuated three times in the intervention year, delaying implementation of the intervention). Lack of exposure of community members to components of the community tobacco intervention may also have played a role; exposure to individual components are reported elsewhere.

Finally, aspects of the research design may have influenced the study's findings. Self-selection of intervention communities meant that there might have been other factors apart from the intervention itself which meant that tobacco consumption was likely to decline. Randomisation of communities was considered when planning the study but was not acceptable to funding agencies and to those involved in service delivery and thus was abandoned. Randomisation would have overcome difficulties in matching control and intervention communities; such a trial could be considered as a future option, using this study as a pilot. However, such a study design may prove to be impractical in a remote setting, especially with disparate communities, and a study design such as case study evaluation may prove more feasible and useful in determining outcomes of such community interventions. While multi-component interventions are more likely to be effective at a community level in the long term, they may be difficult to evaluate in the short term because of the varying effects of component interventions and the time required for them to result in behaviour change in individuals. Other shortcomings of the study design used included that the findings from this small study might not be generalisable to other Aboriginal communities.

Using data on tobacco orders obtained from wholesalers was a quick, cheap and non-invasive method of collecting information. However, there may have been other sources of tobacco in these communities (for example, tobacco bought on visits to regional centres) that was not recorded in turnover from the vendors included in the analysis. This was evident in the discrepancy between tobacco orders and self-reported consumption at the follow-up visit; this may have resulted in an over-estimation of the overall drop in tobacco consumption.

Poor recruitment rates and poor follow-up rate (which appeared to be caused by high mobility of community populations) may have resulted in selection bias in participants in the community sample.

\section{Conclusions}

A multi-component, community-based tobacco intervention delivered in three remote Aboriginal communities appeared to result in a decrease in tobacco consumption (measured through store orders of tobacco) in one intervention community, and a non-significant trend towards decline in consumption compared with control communities. No significant changes in the prevalence of tobacco use were seen, although participants did display increases in readiness to quit and in knowledge about tobacco. As for other populations, multi-component community tobacco interventions are likely to require more research and evaluation; it is recommended that more intensive interventions be trialled.

\section{Acknowledgements}

Funding for this project was provided by the Cooperative Research Centre for Aboriginal and Tropical Health, Territory Health Services, and from the National Health and Medical Research Council. Project workers included Floyd Brooks, Lance George, Ralph Forbes, Juanita Rrupana, John Morgan, (Dorothy) Muwalkmuwuy and (Daphne) Nimadydja. The authors would like to thank the communities that participated in the project and their community councils and health boards.

Lidia Tsirogianis, of Independent Grocers (Darwin), kindly provided information on tobacco orders from participating communities (with their permission). The Institute for International Health, University of Sydney, provided access to office space, and Sam Coleman of IIH provided assistance with statistical calculations.

\section{References}

1. Ivers RG. A review of tobacco interventions for Indigenous Australians. Aust N Z J Public Health. 2003;27(3):294-9.

2. Sowden A, Arblaster L. Community interventions for preventing smoking in young people (Cochrane Review). In: The Cochrane Database of Systematic Reviews, Issue 4, 2000. Oxford (UK): Update Software; 2001.

3. Community Intervention Trial for Smoking Cessation (COMMIT): I. Cohort results from a four-year community intervention. Am J Public Health. $1995 ; 85(2): 183-92$.

4. Community Intervention Trial for Smoking Cessation (COMMIT): II. Changes in adult cigarette smoking prevalence. Am J Public Health. 1995;85(2): 193-200.

5. Hancock L, Sanson-Fisher R, Perkins J, Girgis A, Howley P, Schofield M. The effect of a community action intervention on adolescent smoking rates in rural Australian towns: the CART project. Cancer Action in Rural Towns. Prev Med. 2001;32(4):332-40.

6. National Tobacco Campaign. Australia's National Tobacco Campaign: Evaluation Report Volume 1. Canberra (AUST): Commonwealth Department of Health and Aged Care; 1999. p. 25-45, 240.

7. Australian Bureau of Statistics. 1996 Census of Population and Housing - Aboriginal and Torres Strait Islander People. Canberra (AUST): ABS; 1998.

8. COMMIT Research Group. Community Intervention Trial for Smoking Cessation (COMMIT): II. Changes in adult cigarette smoking prevalence. Am J Public Health. 1995;85(2):193-200.

9. Watson C, Fleming J, Alexander K. A Survey of Drug Use Patterns in Northern Territory Aboriginal Communities 1986-1987. Darwin (AUST): Northern Territory Department of Health and Community Services; 1988.

10. Goto Y. Store Food: A Case Study of the Food Supply in Maningrida from 1988-1995. Canberra (AUST): Australian National University; 1998.

11. STATA: statistical software [computer program]. Version 6.0. College Station (TX): Stata Corporation; 1999.

12. Australian Institute of Health and Welfare. National Drug Strategy Household Survey: Urban Aboriginal and Torres Strait Islander Peoples Supplement 1994. Canberra (AUST): AIHW; 1994.

13. Mullins R, Borland R. Key Findings of the 1993 Household Survey. Melbourne (AUST): Victorian Smoking and Health Program; 1995. Quit Evaluation Studies No.: 7 . 